Revista de Negócios_ISSN 1980.4431_vol. 18, n.3, p.76_94, 2013_DOI:10.7867/1980431.2013v18n3p76_94

\title{
A influência da estratégia e das capabilidades para obtenção de desempenho nas empresas de bens de capital
}

\section{The influence of the strategy and capabilities for obtaining business performance in capital goods companies}

\author{
Roberto Giro Moori \\ Universidade Presbiteriana Mackenzie - Brasil \\ roberto.g.moori@gmail.com \\ Fabio Ytoshi Shibao \\ Universidade Nove de Julho - Brasil \\ fabio.shibao@gmail.com \\ Mario Roberto dos Santos \\ Universidade Nove de Julho - Brasil \\ mario.rsantos@terra.com.br
}

Recebido em 14 de agosto de 2012. Alterado em 15 de março de 2013. Aprovado em 27 de março de 2013

Editor Responsável: Edson Roberto Scharf, Dr.

Processo de avaliação por double blind review

\section{Resumo}

O objetivo deste estudo foi analisar a importância da relação entre estratégias e capabilidades para o desempenho nas empresas do setor de bens de capital. A linha de pensamento teórica para a construção do modelo foi fundamentada em estratégias empresariais, gestão de operações, logística e cadeia de suprimentos. Para a coleta de dados, utilizou-se de questionários semi-estruturado aplicados junto aos gestores de operações, logística e cadeia de suprimentos, de empresas do setor de bens de capital. Os dados coletados foram tratadas pela estatística multivariada, o método da modelagem de equações estruturais, cuja estimação das variáveis latentes (ou construtos) se deu pela máxima verossimilhança. Os resultados mostraram, de um total de 35 medidas, 21 foram validadas, sendo sete relacionadas ao construto Estratégia, dez ao construto Capabilidade e quatro ao Desempenho. Quanto às relações estruturais do modelo, do total de três, duas foram validadas: a relação estratégia / capabilidade e capabilidade / desempenho; e uma, dada pela relação estratégia / desempenho não foi validada. Colocando de outro modo, o estudo mostrou que existe evidência de que a estratégia influencia as capabilidades operacionais, por sua vez, impacta no desempenho das empresas do setor de bens de capital. Não obstante, o desempenho não é influenciado diretamente pela estratégia empresarial. Por conta destes resultados pode-se concluir que o desempenho nas empresas do setor de bens de capital é mais efetivo, quando ajustado à relação entre estratégia e capabilidade, denotando a importância das capabilidades operacionais internas na obtenção da vantagem competitiva.

Palavras-chave: estratégias empresariais, capabilidades operacionais de manufatura, desempenho de 
A influência da estratégia e das capabilidades para obtenção de desempenho nas empresas de bens de capital

negócios, empresas de bens de capital, capabilidades dinâmicas.

\section{Abstract}

The aim of this study was to analyze the importance of the relationship between strategy and performance capabilities to companies in the capital goods sector. The line of thinking to build of the theoretical model was based on business strategies, operations management, logistics and supply chain. For data collection, we used a semi-structured interview and carried with the managers of operations, logistics and supply chain of companies' capital goods sector. The collected data were processed by multivariate analysis, the method of structural equation modeling, whose estimation of latent variables (or constructs) was given by maximum likelihood. The results showed a total of 35 measures, 21 were validated, seven related to construct Strategy, ten to construct Capability and four the Performance' construct. Regarding the structural relationships of the model, the total of three, two have been validated: the relationship strategy / capability and capability / performance, and the relationship strategy / performance have not been validated. In other way, the study showed that there is evidence that the strategy influences the operational capabilities, on the other hand, impacts the companies' performance in the capital goods sector. Nevertheless, the performance is not directly influenced by the business strategy. Because of these results it can be concluded that the performance in the business sector of capital goods is more effective when adjusted the relationship between strategy and capability, emphasizing the importance of internal operational capabilities in obtaining competitive advantage.

Keywords: business strategy, operational capabilities, business performance, capital goods companies, dynamic capabilities.

\section{Introdução}

Em ambiente com altos níveis de incerteza para investimentos gerados por mudanças na economia têm influenciado as empresas a ajustar suas estratégias para identificar oportunidades e explorá-las para a competitividade. Uma das alternativas para esse novo ambiente é a inovação. Inovação não no sentido tecnológico, mas de design, de distribuição, exemplifica Ferraz (2008), que na repaginação das sandálias havaianas, ao ganhar novos desenhos abre oportunidades de se vender em lojas dantes inacessíveis.
Neste caso, investir em inovação, de um lado, aumenta a capacidade das empresas de bens de capital para a fabricação de máquinas e equipamentos; de outro, a capacidade das empresas fabricantes de sandálias para a aquisição de máquinas e equipamentos. Nesse sentido, argumentam Arbix e Salerno (2008), a inovação é apresentada como um processo essencial para gerar e sustentar crescimento econômico e desenvolvimento social, tanto nos países avançados como nos emergentes.

Todavia, em um ambiente competitivo, o consumidor poderá desejar, além de preço menor, outros aspectos de valores agregados ao produto como qualidade e flexibilidade de troca. Considerar cada um desses aspectos pode contribuir para uma posição estratégica de produtos de custo relativo e criar a base para a diferenciação (CHRISTOPHER, 1997), além de provocar alterações na maneira de condução dos negócios. Exemplo disso são as atividades de terceirizações e de cadeias de suprimentos globais em que as pressões por eficiência passaram a recair sobre atividades, cuja competitividade, é determinada na maneira como estas se relacionam e formam uma cadeia de valores (MALLABY, 2007; PORTER, 1985).

Nesse contexto, a ideia de valor pode ser estendida por toda cadeia, viabilizando assim, que os aspectos relevantes (ou prioridades competitivas) desejados pelos consumidores possam ser considerados nas diretrizes estratégicas da empresa. Todavia, o ambiente dinâmico e instável em que o cliente está inserido, levam as empresas a adequar suas diretrizes estratégicas, por consequência, mudar rapidamente as capabilidades da empresa (TEECE; PISANO; SHUEN, 1997). Nesse sentido, a empresa competitiva não é aquela que tem determinados recursos, e sim, aquela que consegue mobilizá-los, em momento oportuno, sob a forma de conhecimentos, relacionamentos e organização e empregá-los na agregação 
de valor a seus produtos.

Drnevich e Kriauciunas (2011) explicitam que pesquisas foram realizadas para verificar a relação entre o dinamismo do ambiente empresarial e a contribuição das capabilidades para o desempenho da empresa, mas que essas relações não foram relativamente testadas.

Assim, dado a necessidade do desenvolvimento de uma gestão empresarial que relacione as diretrizes estratégicas com as capabilidades dinâmicas das atividades produtivas e obter desempenhos operacionais e financeiros consistentes, a questão básica colocada para esta pesquisa foi a seguinte:

Qual é a efetividade da relação entre estratégias e capabilidades para o desempenho nas empresas do setor de bens de capital?

O objetivo geral foi o de analisar a importância da relação entre estratégias e capabilidades para o desempenho nas empresas do setor de bens de capital, a partir do modelo teórico da modelagem em equações estruturais composto pelos construtos: estratégia, capabilidade e desempenho.

Do objetivo geral da pesquisa, foram derivados os objetivos específicos:

a) validar as medidas e escalas do modelo de mensuração do relacionamento entre estratégias, capabilidades e desempenho e;

b) validar as relações estruturais do modelo de mensuração.

O trabalho encontra-se dividido em cinco seções. Na primeira seção é apresentada a introdução complementada pelo problema de pesquisa e objetivos. O modelo conceitual e hipóteses são apresentados na segunda seção. Na terceira, são apresentados os procedimentos métodológicos. A análise dos dados e resultados são apresentados na quarta seção e na quinta seção são apresentadas as conclusões e as sugestões para prosseguimento das pesquisas.

\section{Modelo conceitual e hipóteses}

No artigo seminal de Wood e Zuffo (1998), foi definida a cadeia de suprimentos como fonte de alinhamento de todas as atividades de produção de forma sincronizada, para redução de custos, à minimização dos ciclos e a maximização do valor percebido pelo cliente final por meio do rompimento das barreiras entre departamentos e áreas.

No ambiente corporativo, a busca do alinhamento das estratégias competitivas da empresa com o ambiente externo leva, segundo Brown e Blackmon (2005), a empresa a obter uma vantagem competitiva que gera sinergia de ações positivas nos diversos níveis hierárquicos e departamentais, desde a sua formulação nos níveis mais altos da hierarquia até a camada mais baixa de uma empresa. Acrescenta ainda Morash (2001), é importante considerar os objetivos e valores da empresa nas estratégias da cadeia de suprimentos, capabilidades internas e desempenho. Nesse sentido, para analisar a importância da relação entre estratégias e capabilidades para o desempenho nas empresas do setor de bens de capital, estabeleceu-se o modelo conceitual mostrado na Figura 1. 
A influência da estratégia e das capabilidades para obtenção de desempenho nas empresas de bens de capital

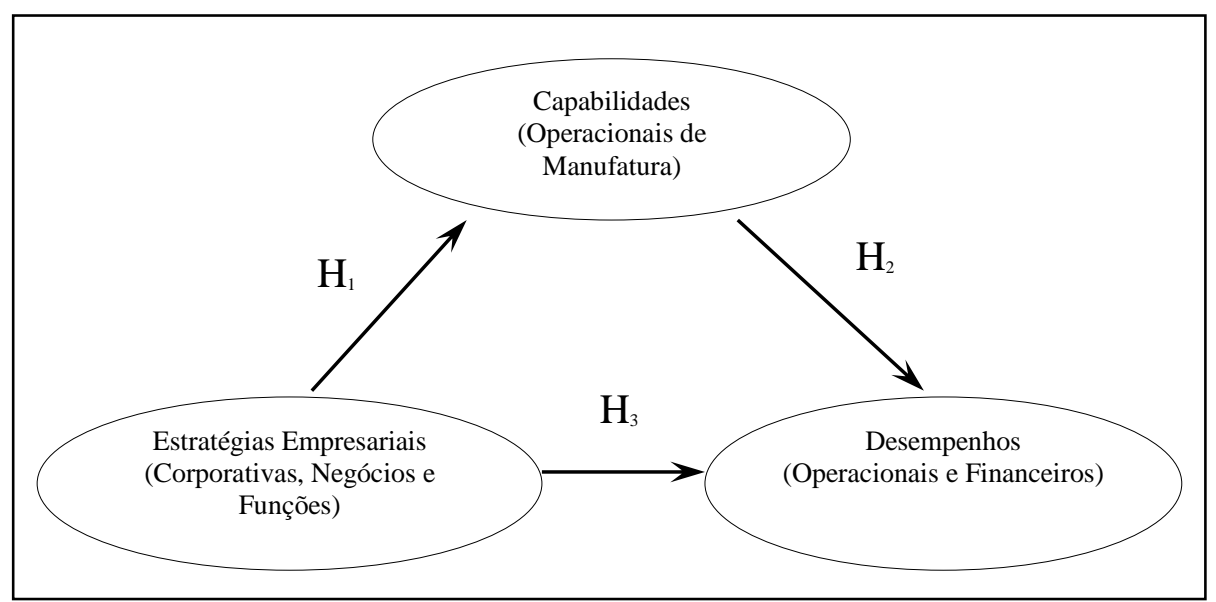

Figura 1: Modelo teórico proposto Fonte: Os autores

As discussões teóricas e hipóteses que suportaram o modelo, estruturado segundo o conceito da relação de causa e efeito, em que as variáveis latentes (ou construtos) se alternam, ora sendo utilizadas como variáveis latentes dependentes, ora como variáveis latentes independentes são apresentadas a seguir.

\subsection{Estratégias empresariais e capa- bilidades operacionais de manufatura}

O construto estratégias empresariais está em lidar com a competição. Nesse sentido, Porter (1985) defendeu que uma empresa pode obter vantagem competitiva por meio de três caminhos: liderança em custo, em diferenciação e em enfoque ou nicho de mercado. No entanto, ressalta Porter (1985), os caminhos são excludentes. Não há como ter um produto diferenciado a baixo custo e vice-versa. Autores como Hunger e Wheelen (2000) seguem uma linha de pensamento antagônica a de Porter (1985) ao citarem, por exemplo, os casos de empresas Japonesas como a Toyota que por meio de seu modelo JIT (Just in Time) e Lean Manufacturing (produção enxuta) é capaz de trabalhar de forma conjunta com ambas estratégias de custo e diferenciação, obtendo dupla vantagem competitiva.
Observa-se assim, embora a cada ano são propostas e difundidas internacionalmente novas teorias ou abordagens sobre gestão estratégica, não há um consenso sobre elas (WHITTINGTON, 2001). A gestão estratégica, que no passado, se resumia às questões econômicas como competitividade, eficiência e lucratividade, atualmente, passou a abarcar assuntos como o meio ambiente, responsabilidades sociais e inovações tecnológicas (BESANKO et al., 2006).

Nas empresas de manufatura, a utilização das capacidades produtivas é de vital importância para a competitividade (GAITHER; FRAZIER, 2001). Isso requer a vinculação das estratégias de manufatura com outras estratégias funcionais como as de marketing, finanças e gestão de pessoas para que elas sejam bem sucedidas. Por conta disso, as estratégias de manufatura devem estar sincronizadas com o ambiente externo por meio da estratégia de negócios (WARD et al., 1995).

Portanto, formular estratégia de manufatura como parte da estratégia de negócios unifica a empresa que se consolida pela estratégia corporativa (KAPLAN; NORTON, 2006). Nessa concepção, a empresa não tem clientes nem opera processos que geram produtos e serviços. Clientes e processos operacionais pertencem ao domínio das unidades de 
negócios. A empresa sincroniza as atividades que criam valor, executadas pelas unidades de negócios, para produzir benefícios para seus clientes ou que reduzam seus custos operacionais totais, além do que poderiam alcançar por si mesmas, se funcionassem com total independência. Mais recentemente, as estratégias de manufatura têm oferecido importantes contribuições para a estratégia corporativa. Por exemplo, Gagnon (1999) considera que a estratégia de manufatura tem papel fundamental nos enfoques baseados em competência e que é possível anular as noções de trade-offs competitivos na formulação de estratégia. É encontrar meios inteligentes para transformar tradeoffs em trade-ons como obter, simultaneamente, oportunidades de melhorar a qualidade e reduzir custos (DEMING, 1990) ou implementar a produção enxuta (lean production) e a agilidade de entrega (CHRISTOPHER; TOWILL, 2001).

Entretanto argumentam Hitt, Ireland e Hoskisson (2002), no âmbito dos negócios, nenhuma estratégia é universalmente superior às outras. A eficácia de cada estratégia depende das oportunidades e ameaças que possam existir no ambiente externo da empresa e das possibilidades proporcionadas pelos recursos e competências da empresa (PRAHALAD; HAMEL, 1990).

O construto capabilidades operacionais de manufatura repousa na visão baseada em recursos (WERNEFELT, 1984) que foi estendido por Teece, Pisano e Shuen (1997) para capabilidades dinâmicas. Segundo Helfat e Winter (2011) algumas definições de capabilidades somente são classificadas como dinâmicas se promoverem grandes mudanças em um curto período de tempo. Acrescentam ainda Helfat e Winter (2011), os recursos que promovem mudanças economicamente importantes, mesmo que gradual são, de fato, também dinâmicas.

As capabilidades dinâmicas são importantes em ambientes de mudanças rápidas e competitivas, cujos produtos ou resultados de qualquer desenvolvimento de produto tem vida mais curta do que em ambiente estável. Estas capabilidades devem ser aproveitadas continuamente para criar, ampliar, atualizar, proteger e manter os ativos relevantes e únicos da empresa (TEECE, 2007). A administração deve ser capaz de adaptar a estratégia, a fim de possibilitar que as condições de estabilidade sejam recriadas. Exemplo, se o ambiente externo deseja pontualidade na entrega de seus produtos adquiridos, a empresa deve atendê-lo.

Para Lynch, Keller e Ozment (2000) estratégias e capabilidades devem estar integradas para atingir desempenho superior. Não obstante, melhorar as capabilidades operacionais dinâmicas exige decisões estratégicas em investimentos direcionados para capacitação gerencial, softwares ou em ativos produtivos como a reformulação de linhas de produtos, adoção de tecnologias de fabricação e de logística. Assim é de se supor que:

$\mathrm{H}_{1}$ : Existe relação positiva entre as estratégias empresariais e as capabilidades operacionais de manufatura.

\subsection{Capabilidades operacionais de manufatura e desempenhos operacionais e financeiros}

O construto capabilidades operacionais de manufatura precisa ser sustentado por processos que asseguram um fluxo constante de melhorias de produtos e pela capacidade de levá-los para o mercado rapidamente. Apenas recursos (BARNEY, 1991; RUMELT, 1991; WERNERFELT, 1984) e competências essenciais (PRAHALAD; HAMEL, 1990) não asseguram que uma empresa possa sustentar sua vantagem. Uma vantagem competitiva é sustentável quando ela persiste apesar dos esforços das empresas concorrentes ou entrantes em potencial para imitá-las ou neutralizá-las. 
A influência da estratégia e das capabilidades para obtenção de desempenho nas empresas de bens de capital

$\mathrm{Na}$ teoria baseada em recursos (Resource-Based View - RBV), segundo Peteraf (1993), a heterogeneidade de recursos é de importância fundamental para a vantagem competitiva. Acrescentam ainda Weerawardena e Mavondo (2011), é um arcabouço teórico que explica a diferença de desempenho das empresas. A RBV destaca que se todas as empresas de um mercado possuem a mesma quantidade de recursos e competências, nenhuma estratégia para a criação de valor estaria disponível para uma empresa sem também estar disponível para todas as outras empresas do mercado. Qualquer outra empresa poderia imediatamente replicar a estratégia que confere vantagem, que segundo Dierickx e Cool (1989) a sustentabilidade depende do quão fácil, os ativos podem ser substituídos ou imitados. Para ser sustentável, uma vantagem competitiva deve então ser baseada em recursos e competências que são escassos e imperfeitamente móveis.

Restrições legais, insumos escassos são barreiras tangíveis à imitação. Não obstante, competências organizacionais distintivas podem ser barreiras intangíveis à imitação. Entre as barreiras intangíveis, Rumelt (1991) destaca:

a) a ambiguidade causal como uma consequência das competências organizacionais distintivas e envolver conhecimento tácito;

b) dependência de circunstâncias históricas como distinção ligada ao histórico da empresa e;

c) complexidade social como uma derivação de processos socialmente complexos.

Nesse sentido, o construto desempenhos operacionais e financeiros para teoria neo-institucional, é uma abordagem do campo de estudos organizacionais que contrapõe os modelos racionais (DiMAGGIO; POWELL, 2005; MEYER; ROWAN, 1991), as organizações não investem necessariamente em modelos de negócios, ferramentas eletrônicas de integração, treinamentos em capacitação gerencial e estruturas produtivas por estas serem as mais eficientes, mas porque adotá-las torna-se fator fundamental para a obtenção de legitimidade em um setor produtivo (MOTTA; VASCONCELOS, 2002).

No entanto, a abordagem das capabilidades operacionais dinâmicas enfatiza que a vantagem competitiva é obtida pelos processos internos da empresa, cujas rotinas, melhoradas continuamente, são capazes de serem adaptadas à dinâmica do mercado. Empresas com capabilidades dinâmicas limitadas, deixam de promover e adaptar as fontes de sua vantagem competitiva ao longo do tempo. Outras, eventualmente as suplantam. Empresas com fortes capabilidades dinâmicas adaptam seus recursos e competências essenciais ao longo do tempo e tiram vantagem das novas oportunidades de mercado para criar novas fontes de vantagem competitiva (TEECE; PISANO; SHUEN, 1997), isto é, precisam reconfigurar suas capabilidades para enfrentar os desafios na rápida evolução dos negócios (WEERAWARDENA; MAVONDO, 2011).

Em geral, as capabilidades operacionais dinâmicas como resultado da combinação de investimentos em recursos tangíveis como as ampliações do parque fabril ou intangíveis, consubstanciadas em treinamento de pessoal, marcas ou patentes, proporcionam relação positiva como os desempenhos operacionais (CHASE; JACOBS; AQUILANO, 2006). Assim é de se supor que:

$\mathrm{H}_{2}$ : Existe relação positiva entre capabilidades operacionais de manufatura e desempenhos operacionais e financeiros.

\subsection{Estratégias empresariais e desem- penhos operacionais e financeiros}

Assim o construto estratégias empresariais envolve um conjunto de atividades, sistemas de informação e 
capacitações gerenciais, cujos resultados, somente são revelados a posteriori. Ainda que, em ambientes turbulentos e de incerteza, as empresas devem se posicionar estrategicamente. As ações dos gestores estratégicos tem que ser coerente com o ambiente, atenuando as condições geradoras de incerteza, de modo a manter a estabilidade dos negócios frente às ameaças do ambiente e, simultaneamente, aproveitar as oportunidades nele identificadas, e assim, obter retornos satisfatórios para seus investimentos.

O construto desempenhos operacionais e financeiros exige das empresas que fazem pesados investimentos para desenvolver novos produtos, para recuperar seus investimentos um grande mercado para que possam reaver os gastos efetuados em pesquisa e desenvolvimento, capacitação gerencial ou tecnologias de processos. Em ambientes de alta turbulência e competitividade, os investimentos tem que ser recuperados de uma forma mais veloz (HITT; IRELAND; HOSKISSON, 2002; ROSS; WESTERFIELD; JORDAN, 2008). Além disso, as capacidades das firmas para desenvolver novas tecnologias estão se expandindo e, devido a diferentes leis de patentes em vários países, a imitação e a prática da engenharia reversa são as estratégias mais prováveis de serem adotadas pelos competidores.

A ampliação de mercado por meio da expansão internacional é atraente para muitas empresas porque ampliam a oportunidade de se recuperar os investimentos em tecnologias de processos e gastos em capacitação gerencial em grande escala. Não obstante, empresas que atuam em ambiente internacional, são mais expostas a dois fatores macroeconômicos: flutuação da taxa de câmbio e inflação (DORNIER et al., 2000).

Nesse sentido, empresas mesmo estando em uma situação de competitividade, e relativa estabilidade nos negócios, fatores macroeconômicos podem alterar condições financeiras, que na busca de proteção dos investimentos efetuados e garantia de taxas de retornos condizentes para seus acionistas, levam empresas a adotar um perfil mais especulativo junto ao mercado financeiro (BACIC; DA SILVEIRA; FERREIRA DE SOUZA, 2010). Abordagens estratégicas direcionadas por mais lucro a prazos mais curto pode conduzir a busca de retorno de investimento pelo lado financeiro por maiores ganhos, levando a uma situação de financeirização da empresa. A financeirização, segundo Bresser-Pereira (2009) é uma distorção do sistema financeiro em que se permitiu, em vários casos, criar riquezas fictícias por meio de taxas de lucro normal das empresas ou ganhos financeiros muito mais elevados do que a taxa de juros ou crescimento do Produto Interno Bruto (PIB).

Observa-se em empresas de manufatura em situação de financeirização, uma separação entre as funções produtivas e financeiras cujas taxas de retornos de investimento são decorrentes muito mais de aplicações financeiras do que de atividades produtivas.

É de se esperar que, em empresas não financeiras, a fonte mais duradora de lucro seja a de produtos e não a aplicação de excedentes, que rigorosamente, deveriam ser destinados a investimentos nas áreas operacionais da empresa, em tecnologia, em inovação, em melhoria de produtos, de forma que possa competir em posição mais favorável. O modelo de gestão com ênfase no lado financeiro pode conduzir a um perigoso descuido com o lado produtivo em favor de aplicações financeiras. Isso vai deteriorando as condições de concorrência da empresa, pois ela vai ficando mais defasada tecnologicamente e, em consequência, em produtos. Além disso, há o próprio risco de apostar na tendência errada (BACIC; DA SILVEIRA; FERREIRA DE SOUZA, 2010).

Nesse aspecto, na década de 1960, Skinner (1969) argumentava que a 
A influência da estratégia e das capabilidades para obtenção de desempenho nas empresas de bens de capital

conexão entre as estratégias corporativas e de manufatura era um elo perdido, muitas vezes, em decorrência das decisões estratégicas nas empresas serem tomadas por indivíduos com pouco conhecimento sobre operações de manufatura, logística ou fornecimento de serviços (BROWN et al., 2006). Assim é de se supor que:

$\mathrm{H}_{3}$ : Existe relação positiva entre as estratégias empresariais e desempenhos operacionais $\mathrm{e}$ financeiros

\section{Procedimentos metodológicos}

Nesta seção é apresentado o método utilizado na pesquisa, a natureza e tipo de pesquisa, instrumento de coleta, amostra e sujeito da pesquisa.

\subsection{O método}

O método utilizado foi $\mathrm{o}$ da modelagem de equações estruturais, inspirado no modelo teórico mostrado na Figura 1. A estratégia de modelagem adotada foi a confirmatória em que se especificou um só modelo teórico (HAIR et al., 2005).

A aplicação da modelagem de equações estruturais, a estratégia confirmatória, foi precedida de uma análise fatorial exploratória, utilizando-se do Método da Máxima Verossimilhança (ML), para a análise dos construtos e suas respectivas variáveis. A ML é um método alternativo aos usuais mínimos quadrados em regressão múltipla, cujo procedimento, melhora por interações as estimativas de parâmetros para minimizar uma função de ajuste especificada. Além disso, dada as propriedades estatísticas da ML e suas características em amostras menores, as análises tendem a serem mais conservadoras (HAIR et al., 2005).

As medidas e escalas do modelo de mensuração obtidas pela análise fatorial confirmatória, em nível de significância ( $\alpha$ $\leq 0,05)$ para a validação:

a) convergente, utilizou-se da
Variância Média Extraída (VME) que reflete a quantia geral de variância nos indicadores explicada pela variável latente. Hair et al. (2005) consideram adequada quando a VME é superior a 0,5 ;

b) discriminante, utilizou-se da análise das variâncias extraídas mais elevadas do que as variâncias partilhadas entre fatores, e expressas pelos quadrados dos respectivos coeficientes de correlação (KLINE, 2005) e;

c) confiabilidade, utilizou-se do Alpha $(\alpha)$ de Cronbach, cujo valor recomendado deve ser maior do que 0,6 (HAIR et al., 2005).

Para validar a estrutura do modelo de mensuração, dentre uma diversidade de índices disponíveis, foram adotados o coeficiente de determinação, $\mathrm{R}^{2}$, e as medidas de ajustes: Goodness of Fit Index (GFI), Adjusted Goodness of Fit Index (AGFI), Parsimony Goodness of Fit Index (PGFI), Root Mean Square Residual (RMR), Root Mean Square Error of Approximation (RMSEA) e $90 \%$ Confidence Interval for RMSEA do software LISREL 8.17.

Limitação do Método: A pesquisa apresentou duas limitações básicas. A primeira referiu-se à amostra. Para a aplicação de técnicas estatísticas multivariadas como a análise fatorial e modelagem em equações estruturais, o mínimo é ter pelo menos cinco vezes mais observações do que o número de variáveis a serem analisadas, e o tamanho mais aceitável é ter uma proporção de 10 para um (HAIR et al., 2005). Estas condições não foram obedecidas, todavia, foram realizados todos os testes sugeridos por Hair et al., (2005) para a aplicação da análise fatorial. Não obstante, a disponibilidade de softwares estatísticos programados para executar rotinas com dados não normal e mesmo de pequeno tamanho, não impedem que esta limitação possa ser contornada. A 
segunda referiu-se a escolha da estratégia de modelagem de equações estruturais. Existem diversas outras estratégias de modelagem como a de modelos concorrentes e a de desenvolvimento de modelos (re-especificação) que poderiam ter ajustes igualmente aceitáveis, cujos testes mais rigorosos são obtidos pela comparação de modelos alternativos. Esta limitação ficou como sugestão para futuras pesquisas. Diante destas limitações, a generalização dos resultados obtidos por este estudo, deve ser feita com ressalva.

Os detalhes dos procedimentos metodológicos relacionados à natureza, tipo da pesquisa, estudo anterior, instrumento de coleta de dados, amostra, sujeitos da pesquisa e delimitação do estudo, são descritos a seguir.

\subsection{Natureza e tipo de pesquisa, instrumento de coleta, amostra e sujeito da pesquisa}

A pesquisa foi considerada de natureza exploratória do tipo explicativa. Exploratória porque em atividades de modelagem é comum testar diversos modelos alternativos. Jöreskog e Sörbom (1993) reforçam este comentário ao citar as aplicações usuais da modelagem em equação estruturais: testar um único modelo teórico; comparar modelos alternativos e gerar modelos mais eficientes, modificados a partir do modelo proposto. Foi explicativa porque a pesquisa visou esclarecer quais variáveis contribuíram de alguma forma, para a ocorrência de determinado fenômeno, que nesta pesquisa, tratou-se da relação entre estratégia, capabilidade e desempenho (VERGARA, 2009).

Para operacionalizar o modelo teórico, a pesquisa foi precedida de uma pesquisa, de natureza exploratória, composta de entrevistas em profundidade e focus group com 10 especialistas junto às empresas do setor de bens de capital, correspondente aos grupos da
Classificação Nacional de Atividades Econômica (IBGE/CNAE): 25 a 36, envolvendo os fornecedores e fabricantes de máquinas e equipamentos (IBGE/CNAE, 2011). Ao final, foram geradas 100 assertivas, distribuídas nas três categorias: estratégia, capabilidade e desempenho.

Todavia, as assertivas geradas foram consideradas excessivas para a construção do questionário. Para reduzi-las e atingir um número razoável, realizou-se pré-testes do instrumento de coleta junto a 22 respondentes para avaliar o conteúdo, a quantidade e distribuição das assertivas bem como o formato estético do questionário. Mediante a incorporação das sugestões, restaram 35 assertivas sendo: oito assertivas para o construto estratégia (corporativa, negócios e funções), 19 assertivas para as capabilidades (manufatura e logística) e oito assertivas para desempenho (financeiro e logístico).

Definidas as assertivas, construiuse um questionário estruturado composto de perguntas fechadas e abertas, composto de seis blocos de respostas. O primeiro bloco referiu-se aos dados do respondente. O segundo bloco relacionou-se aos dados da empresa. Nos três blocos seguintes, de número três, quatro e cinco foi solicitado ao respondente que assinalasse com um ' $x$ ', na assertiva e respectivo grau de concordância, que variava entre Discordo Totalmente $(\mathrm{DT}=1)$ e Concordo Totalmente $(\mathrm{CT}=6)$, sendo que o bloco três, constituído de 8 assertivas, referiu-se às estratégias; 0 bloco quatro foi constituído de 19 assertivas e referiu-se às capabilidades e; o bloco cinco referiu-se ao desempenho. Ressalta-se que neste bloco, para a percepção do desempenho em relação à assertiva apresentada, o respondente deveria se basear aos últimos três anos, apresentando assim, características de concepção longitudinal na coleta de dado deste bloco. Por fim, o bloco seis, foi constituído de uma pergunta aberta, em que foi solicitado ao 
A influência da estratégia e das capabilidades para obtenção de desempenho nas empresas de bens de capital

respondente, comentários adicionais, caso o considerasse relevante.

Definido o questionário escolheu-se uma amostra de empresas a partir do cadastro de contato de uma empresa de consultoria localizada na cidade de São Paulo, SP. Tendo como base o ramo de atividade das empresas contidas no cadastro e a função dos respondentes como diretores, gerentes e profissionais relacionados à operação, logística e de cadeia de suprimentos como potenciais respondentes, em 2008, os questionários foram enviados pelo correio, juntamente com um envelope pré-pago, bem como, disponibilizado em uma página de internet (html) e enviadas mensagens, via correio eletrônico (e-mail) sobre a pesquisa, juntamente com uma senha de acesso no endereço web em que se solicitava aos potenciais respondentes responder ao questionário.

Delimitação do Estudo. A principal delimitação referiu-se à concepção transversal da pesquisa, em que se retiraram dados de uma amostra fixa de empresas do setor de bens de capital uma única vez. De maneira geral, segundo Easterby-Smith, Thorpe e Lowe (1999) os estudos de concepções transversais, particularmente quando usam questionários para a coleta de dados, descrevem as características de relação de estratégia, capabilidades e desempenho de um grande número de empresas, de portes diferentes e contextos variados, mesmo delimitados ao setor de bens de capital. Por conta disso, fica difícil eliminar todos os fatores externos que poderiam ter causado a relação observada, por consequência, explicar a relação de causa e efeito.

Para contornar esta delimitação, foi sugerido como prosseguimento, realizar a pesquisa em concepção longitudinal. Assim, em um pequeno número de empresas durante períodos prolongados, será possível identificar os processos de mudanças dentro de um contexto mais amplo como social, econômico e político, que circunda a empresa.

\section{Análise dos dados e resultados}

Os dados foram coletados em 2008. Do total de 721 questionários enviados, consideraram-se válidos, 12,5\%, ou seja, 90 questionários. As análises são descritas a seguir.

\subsection{Aspectos demográficos da amostra}

Os dados demográficos relativos aos respondentes e as empresas aqui pesquisadas são assim constituídos:

a) Em relação aos respondentes: Os cargos de diretores, gerentes e supervisores relacionados às atividades de operações e logística representaram $48,7 \%$ das respostas e $51,3 \%$ como encarregados e coordenadores. A formação acadêmica dos respondentes, a administração foi predominante com $55,5 \%$ do total, seguido pela formação em engenharia com $21,7 \%$. O tempo na função e na empresa, acima de cinco anos, os respondentes apresentaram os valores de $48,9 \%$ e $37,8 \%$, respectivamente.

Em resumo: $\mathrm{O}$ perfil dos respondentes foi caracterizado por funções em posição hierárquica de liderança, com formação em administração, mão de obra qualificada, oriundas de diversas áreas do conhecimento e de baixa rotatividade com tendência à renovação.

b) Em relação às empresas: $54,4 \%$ dos respondentes eram oriundos dos fabricantes de máquinas e equipamentos e $45,6 \%$ do total, pertenciam ao ramo metal mecânico como as empresas fabricantes de rolamentos, motores elétricos, autopeças, transformadores, geradores e fundição.

Quanto à localização, a amostra 
apresentou uma concentração de empresas sediadas no Estado de São Paulo com $83,4 \%$ do total de empresas. O número de empregados, segundo o critério do Serviço Brasileiro de Apoio às Micro e Pequenas Empresas (SEBRAE, 2011), 5,6\% foi constituído de empresas com até 19 empregados, $24,4 \%$ entre 19 e 99 empregados, $31,1 \%$ entre 100 e 499 empregados e acima de 500 empregados correspondeu a $38,9 \%$ das empresas respondentes.

Quanto ao porte, segundo o critério do Banco Nacional de Desenvolvimento Econômico e Social (BNDES, 2010), o faturamento anual menor ou igual a $\mathrm{R} \$ 2,4$ milhões, correspondeu a $12,5 \%$ das empresas, $22,7 \%$ correspondeu ao faturamento anual entre $\mathrm{R} \$ 2,4$ e $\mathrm{R} \$ 16,0$ milhões, $23,9 \%$ entre $\mathrm{R} \$ 16,0$ e $\mathrm{R} \$ 90,0$ milhões e 40,9\% acima de $\mathrm{R} \$ 90,0$ milhões. As vendas, em termos médios porcentuais, $54,2 \%$ do total das empresas dependiam do mercado interno, correspondente a faturamento acima de $80 \%$. Em termos de exportação, apenas $5 \%$ das empresas entrevistadas tinham faturamento acima de $80 \%$ dependente do mercado externo.

Em resumo: A amostra revelou predominância de empresas do ramo de metal mecânico e fornecedoras para os fabricantes de máquinas e equipamentos com 54,4\% do total, sediadas no Estado de São Paulo, com produção sob pedido, grande porte e faturamento dependente do mercado interno.

\subsection{Validação das medidas e escalas}

Para obter uma adequada unidimensionalidade e ser coerente com o modelo teórico, utilizou-se da análise fatorial confirmatória para extrair três fatores principais, representados pelos construtos: Estratégia, Capabilidade e Desempenho.

Após a obtenção de uma série de matrizes de correlações pelo método de extração da Máxima Verossimilhança (ML
- Maximum Likelihood), Eigenvalue (autovalor) maior ou igual a um, rotação Varimax e normalização Kaizer para melhorar a interpretação dos constructos latentes, de um total de 35 indicadores do modelo inicial, 16 foram eliminados. Assim, os 21 indicadores remanescentes, foram distribuídos da seguinte forma:

a) Construto Estratégia, sete indicadores: E1 (Integração de setores), E2 (Rapidez no atendimento), E3 (Qualidade na fonte), E4 (Tecnologia de processo), E5 (Otimização de layout), E6 (Manufatura enxuta) e E8 (Orientada para o nível de serviço).

b) Construto Capabilidade, 10 indicadores: $\mathrm{C} 7 \quad$ (Estratégias distintas), C8 (Orientada para o nível de serviço), C11 (TPM - Total Productive Maintenance), C12 (4 Vs - Volume, Variedade, Variação e Visibilidade), C14 (Atividades de pequenos grupos), C15 (Integração da informação), C16 (Processo suportado por TI), C17 (Gestão de processos), C18 (Redução da variabilidade) e C19 (Treinamento de colaboradores) e

c) Construto Desempenho, quatro indicadores: D1 (Custo logístico), D2 (Lucratividade), D3 (Tempo de entrega) e D4 (Tempo de processamento).

A medida de adequação da amostra de Kaiser-Meyer-Olkin (KMO) representada pelo índice (MSA/KMO) foi igual a 0,892, indicando a adequação dos dados para a aplicação da análise fatorial. A análise foi considerada adequada porque apresentou uma variância explicada de $59,9 \%$, valor próximo ao mínimo recomendado de $60 \%$.

Na Tabela 1 são mostrados os resultados da análise fatorial confirmatória. 
A influência da estratégia e das capabilidades para obtenção de desempenho nas empresas de bens de capital

Tabela 1: Análise Fatorial Confirmatória - ML (Maximum Likelihood)

\begin{tabular}{|c|c|c|c|c|c|}
\hline \multirow{2}{*}{\multicolumn{2}{|c|}{ VARIÁVEIS }} & \multicolumn{3}{|c|}{ Maximum Likelihood } & \multirow{2}{*}{$\begin{array}{c}\text { Comuna } \\
\text { lidade }\end{array}$} \\
\hline & & 1 & 2 & 3 & \\
\hline E1 & Integração de setores & 0,095 & 0,559 & 0,370 & 0,459 \\
\hline E2 & Rapidez no atendimento & 0,214 & 0,656 & 0,035 & 0,478 \\
\hline E3 & Qualidade na fonte & 0,317 & 0,761 & 0,116 & 0,693 \\
\hline $\mathrm{E} 4$ & Tecnologias de processo & 0,257 & 0,761 & 0,203 & 0,686 \\
\hline E5 & Otimização de layout & 0,356 & $\mathbf{0 , 5 3 2}$ & 0,303 & 0,502 \\
\hline E6 & Manufatura enxuta & 0,442 & 0,601 & 0,086 & 0,564 \\
\hline E8 & Sincronia dos processos & 0,288 & 0,699 & 0,235 & 0,626 \\
\hline $\mathrm{C} 7$ & Estratégias distintas & $\mathbf{0 , 5 3 8}$ & 0,480 & 0,214 & 0,567 \\
\hline $\mathrm{C} 8$ & Orientada para o nível de serviço & 0,604 & 0,276 & 0,360 & 0,571 \\
\hline $\mathrm{C} 11$ & TPM (Total Productive Maintenance) & $\mathbf{0 , 6 5 9}$ & 0,264 & 0,142 & 0,525 \\
\hline $\mathrm{C} 12$ & 4 Vs (Volume, Variedade, Variação e Visibilidade) & 0,712 & 0,276 & 0,288 & 0,666 \\
\hline $\mathrm{C} 14$ & Atividades de pequenos grupos & 0,742 & 0,157 & 0,162 & 0,601 \\
\hline $\mathrm{C} 15$ & Integração da informação & 0,610 & 0,346 & 0,269 & 0,564 \\
\hline $\mathrm{C} 16$ & Processo suportado por TI & 0,637 & 0,173 & 0,084 & 0,442 \\
\hline $\mathrm{C} 17$ & Gestão de processos & 0,742 & 0,209 & 0,174 & 0,625 \\
\hline $\mathrm{C} 18$ & Redução da variabilidade & 0,746 & 0,301 & 0,274 & 0,723 \\
\hline C19 & Treinamento de colaboradores & 0,742 & 0,391 & 0,130 & 0,720 \\
\hline D1 & Custo logístico diminuiu & 0,310 & 0,244 & 0,482 & 0,388 \\
\hline D2 & Lucratividade aumentou & 0,259 & 0,282 & 0,603 & 0,510 \\
\hline D3 & Tempo de entrega diminuiu & 0,201 & 0,110 & $\mathbf{0 , 8 2 5}$ & 0,733 \\
\hline D4 & Tempo de processamento diminuiu & 0,154 & 0,118 & 0,947 & 0,935 \\
\hline & EIGENVALUE (auto valor) & 5,435 & 4,113 & 3,028 & \\
\hline & Alpha de Cronbach & 0,932 & 0,891 & 0,856 & \\
\hline & AVE (Average Variance Explained) & 25,881 & 19,587 & 14,418 & \\
\hline & AVE Acumulada & 25,881 & 45,468 & 59,886 & \\
\hline & Interpretação (Variáveis Latentes) & $\begin{array}{l}\frac{0}{\tilde{J}} \\
:= \\
:= \\
\tilde{J} \\
\tilde{\tilde{J}} \\
\tilde{U}\end{array}$ & 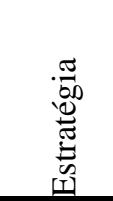 & 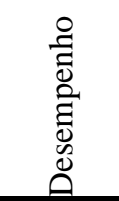 & \\
\hline
\end{tabular}

Kaiser-Meyer-Olkin Measure of Sampling Adequacy: 0,892; $\mathrm{n}=90$ casos; Bartlett's Test of Sphericity -

Approx. Chi-Square: 1345,44; df. 210; Sig. 0,000

Fonte: Dados da pesquisa

Quanto à validação do modelo de mensuração, em relação:

a) à convergente, avaliada pela variância média extraída, o fator estratégia apresentou valor igual a 0,61. Os fatores capabilidade e desempenho apresentaram valores iguais a 0,62 e 070, respectivamente. Estes valores denotaram que o conceito de interesse foi bem representado, dado que, para a variância extraída, o parâmetro de referência é que seja maior que $50 \%$; b) à discriminante, avaliada pelas cargas cruzadas, em todas as correlações, apresentaram valores da raiz quadrada da variância média, superiores à correlação entre os construtos, denotando assim, que dois conceitos similares, são distintos. Na Tabela 2, são mostrados os valores da correlação entre os construtos, e na diagonal, a raiz quadrada da variância média extraída e;

c) á confiabilidade ou consistência interna, avaliada pelo Alpha $(\alpha)$ 
de Cronbach, observou-se que os construtos com suas medidas finais, apresentaram Alpha $(\alpha)$ de Cronbach, maiores do que 0,8 , mostrando assim, que as medidas em seus respectivos construtos são consistentes.

Tabela 2: Correlação entre os construtos: Estratégia, Capabilidade e Desempenho, com a raiz quadrada da variância média extraída na diagonal.

\begin{tabular}{l|c|c|c}
\hline CONSTRUTOS & Estratégia & Capabilidade & Desempenho \\
\hline Estratégia & $\mathbf{0 , 7 7 9}$ & & \\
\hline Capabilidade & 0,691 & $\mathbf{0 , 7 8 9}$ & \\
\hline Desempenho & 0,528 & 0,559 & $\mathbf{0 , 8 3 6}$ \\
\hline
\end{tabular}

Fonte: Dados da pesquisa

Assim, validadas as medidas e escalas do modelo prosseguiu-se na avaliação das relações estruturais do modelo de mensuração.

\subsection{Avaliação das relações estruturais do modelo de mensuração}

Buscou-se, assim, na modelagem de equações estruturais, verificar possíveis discrepâncias ou variações mais específicas no modelo teórico-empírico em análise.

Os dados tratados por meio do software LISREL 8.71, construiu-se o modelo teórico-empirico conforme é mostrado na Figura 2.

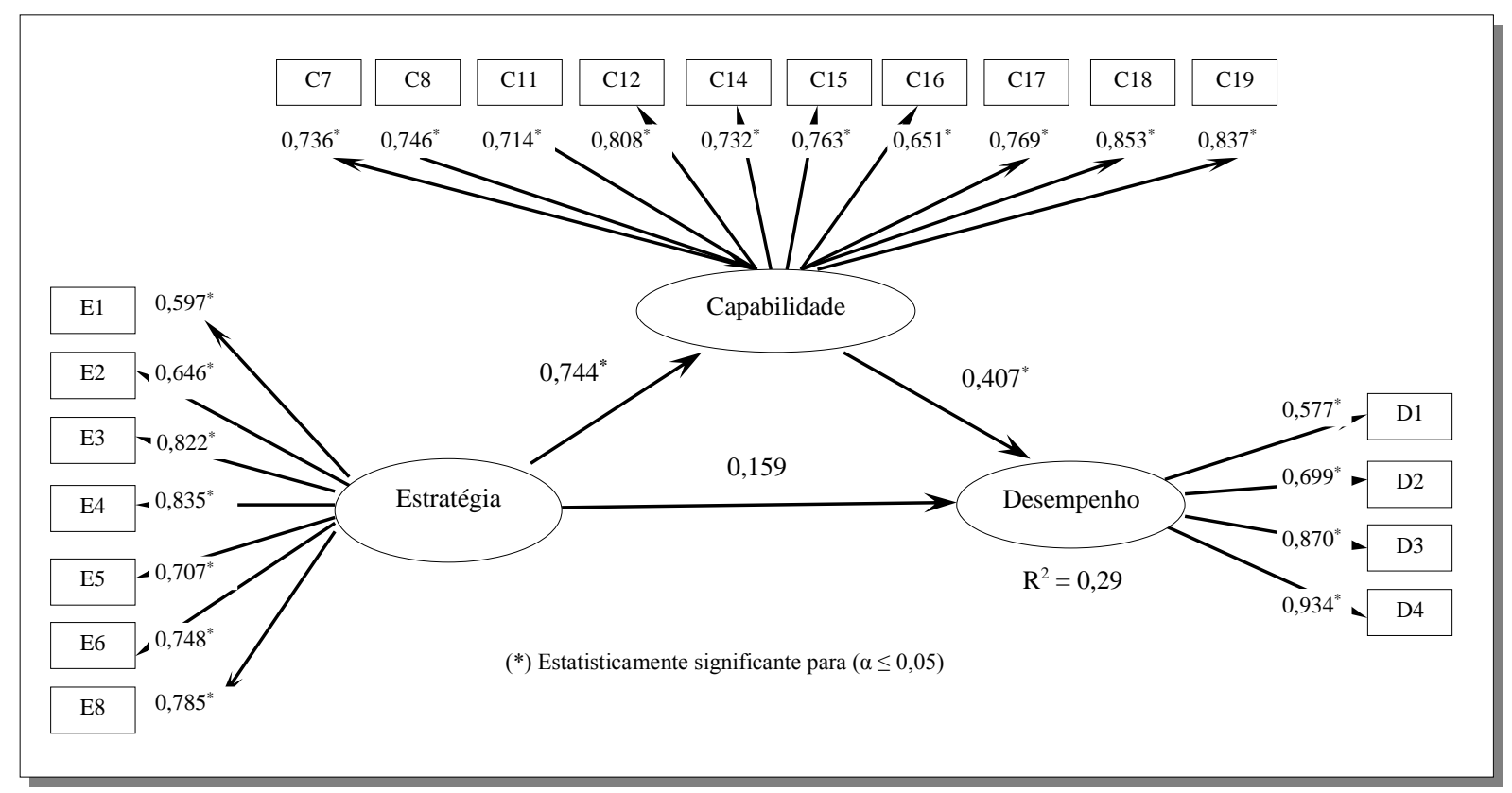

Figura 2: Modelo teórico-empírico (equações estruturais)

Fonte: Dados da pesquisa

Observou-se pela Figura 2 que:

a) o efeito direto [Estratégia $\rightarrow$ Desempenho] foi de 0,159;

b) o efeito indireto [Estratégia $\rightarrow$ Capabilidade $\rightarrow$ Desempenho] foi de $0,744 * 0,407=0,302$; c) o efeito total da estrutura [efeito direto + efeito indireto] foi de 0,159 $+0,302=0,461$

d) o coeficiente de correlação entre os construtos estratégia e desempenho, representado por 0,159, não foi estatisticamente 
A influência da estratégia e das capabilidades para obtenção de desempenho nas empresas de bens de capital

significante.

A eficiência dos estimadores, dado pelo coeficiente de determinação (ou correlação) foi de 0,29, indicando que, aproximadamente, $29 \%$ da variação do desempenho estava relacionada com a variação de capabilidade e de desempenho.
$\mathrm{O}$ fato de $\mathrm{R}^{2}$ não estar próximo de zero sugeriu que a equação foi melhor que a média do desempenho como preditora.

Os principais índices de ajuste, necessários para a avaliação do modelo teórico-empírico, são mostrados na Tabela 3.

Tabela 3: Índices de ajuste (Goodness of fit)

\begin{tabular}{|c|c|c|}
\hline Índices & $\begin{array}{l}\text { Valores } \\
\text { Calculados }\end{array}$ & $\begin{array}{l}\text { Valores Referenciais } \\
\text { (Hair et al., 2005) }\end{array}$ \\
\hline$\chi^{2}$ igual a 327,44 com 186 graus de liberdade & $\mathrm{p} \leq 0,000$ & Significante $(\alpha \leq 0,05)$ \\
\hline Goodness of Fit Index (GFI) & 0,74 & $\geq 0,90$ \\
\hline Adjusted Goodness of Fit Index (AGFI) & 0,68 & $\geq 0,90$ \\
\hline Parsimony Goodness of Fit Index (PGFI) & 0,60 & $0<$ PGFI $<1,0$ \\
\hline Root Mean Square Residual (RMR) & 0,16 & $\leq 0,08$ \\
\hline Root Mean Square Error of Approximation (RMSEA) & 0,092 & $0,05<$ RMSEA $<0,08$ \\
\hline 90\% Confidence Interval for RMSEA & 0,$076 ; 0,11$ & 0,$08 ; 0,10$ \\
\hline
\end{tabular}

Com relação às medidas básica de ajuste do modelo, observou-se que o valor do qui-quadrado $\left(\chi^{2}\right)$ de 327,44 com 186 graus de liberdade foi estatisticamente significante no nível $(\alpha \leq 0,05)$. Examinando outras medidas de ajuste o GFI, AGFI e o RMR estavam abaixo do nível de aceitação. A medida PGFI, igual a 0,6 indicando um ajuste adequado. Como complemento a essas medidas básicas o RMSEA teve um valor de 0,092 , fora do intervalo aceitável de 0,08 ou menos, mas inferior à referência superior de 0,10 .

De forma geral, as medidas de ajustes obtidos indicaram que o modelo foi, na melhor das hipóteses, perifericamente aceitável. Todavia, observou-se que as medidas básicas de ajuste do modelo teórico-empírico forneceram substanciais suportes para explorar possíveis modificações que podem melhorá-lo, por meio de um exame mais cuidadoso dos resultados.

Ponto importante observado foi que na modelagem teórico-empírico em que se utilizou de equações estruturais, o relacionamento entre estratégia e desempenho não foram significantes, evidenciando assim, que não existiu um impacto direto da estratégia sobre o desempenho, denotando a importância das capabilidades para a vantagem competitiva.

Com relação às hipóteses definidas para o estudo e mostradas na Figura 1, na Tabela 4, são mostrados os resultados.

Tabela 4: Coeficientes estruturais do modelo de mensuração e testes de hipóteses

\begin{tabular}{l|c|c|c|c|c}
\hline \multicolumn{1}{c|}{ Relacionamento Estrutural } & $\begin{array}{c}\text { Coeficiente } \\
\text { Estrutural }\end{array}$ & $\begin{array}{c}\text { Erro } \\
\text { Padrão }\end{array}$ & $\begin{array}{c}\text { Valor } \\
\mathbf{t}\end{array}$ & Hipóteses & $\begin{array}{c}\text { Decisão } \\
(\boldsymbol{\alpha} \leq \mathbf{0 5})\end{array}$ \\
\hline Estratégia $\rightarrow$ Capabilidade & 0,744 & 0,12 & 6,13 & $\mathrm{H}_{1}{ }^{* * *}$ & Suporta \\
\hline Capabilidade $\rightarrow$ Desempenho & 0,407 & 0,18 & 2,29 & $\mathrm{H}_{2}{ }^{*}$ & Suporta \\
\hline Estratégia $\rightarrow$ Desempenho & 0,159 & 0,17 & 0,95 & $\mathrm{H}_{3}$ & Não suporta \\
\hline
\end{tabular}

$(* *)<0,01$ : Nível de significância $(\mathrm{t}>2,58) ;(*)<0,05$ : Nível de significância $(\mathrm{t}>1,96)$

Fonte: Dados da pesquisa

Observou-se, portanto, que nos níveis de significâncias estatísticas $\alpha \leq$
0,01 e $\alpha \leq 0,05$, as hipóteses $\mathrm{H}_{1}$ e $\mathrm{H}_{2}$ foram suportadas, respectivamente. Entretanto 
para a hipótese $\mathrm{H}_{3}$ pode-se inferir que existem evidências insuficientes a respeito da relação positiva entre as estratégias empresariais e desempenhos operacionais e financeiros.

Na visão de Cepeda e Vera (2007) algumas definições são prescritivas assumindo que a posse de capabilidades dinâmicas é uma fonte de vantagem competitiva e que elas são tautológicas, isto é, se a empresa tem capabilidades dinâmicas ela deve ter um bom desempenho, e se tem um bom desempenho ela deve ter capabilidades dinâmicas.

A rejeição da hipótese $\mathrm{H}_{3}$ parece estar em acordo com trabalhos similares como o de Green, Whitten e Inman (2008) cujos resultados mostraram que nem gestão estratégica da cadeia de suprimentos nem desempenho logístico impactam diretamente no desempenho financeiro. Colocando de outro modo, o impacto da gestão estratégica no desempenho financeiro ocorre por meio de caminhos indiretos.

\section{Conclusões e sugestões para prosse- guimento}

O estudo mostrou que do total de 35 medidas utilizadas no modelo teórico inicial, 21 foram validadas, sendo sete relacionadas ao construto Estratégia, dez ao construto Capabilidade e quatro ao Desempenho. Quanto às relações estruturais do modelo, do total de três, duas foram validadas: a relação estratégia / capabilidade e capabilidade / desempenho, sendo que a relação estratégia / desempenho não foi validada. Destes resultados, emergiram três implicações teórico-empíricas.

A primeira referiu-se à influência das estratégias empresariais nas capabilidades operacionais. As capabilidades são constituídas de recursos, rotinas e competências difíceis de serem imitadas. Assim, o sucesso da empresa não está em fabricar produtos inovativos, mas em um conjunto único de rotinas, recursos e competências que permitem à empresa fabricar produtos competitivos. Em ambiente de alta competição, o que importa não são apenas os recursos (valioso, raro, inimitável e insubstituível) e as competências essenciais, mas a capabilidade de aperfeiçoá-los e desenvolvê-los continuamente (TEECE; PISANO; SHUEN, 1997).

A segunda referiu-se ao impacto das capabilidades nos desempenhos operacionais e financeiros. Trabalhos como de Tan, Kannan e Narasimhan (2007) demonstram existir evidências de relacionamento positivo entre capabilidades e desempenho, corroborando o resultado obtido. Não obstante, dado as características das empresas de bens de capital como usuário de ativo intensivo, o resultado obtido, evidencia o equilíbrio; de um lado, das necessidades de recuperação dos investimentos realizados pelas empresas em recursos (novas tecnologias), aprimoramento de rotinas e desenvolvimento de competências gerenciais, de modo rápido para em atender demandas de um mercado cada vez mais competitivo e inovativo (HITT; IRELAND; HOSKISSON, 2002). De outro, das pressões por taxas de retorno crescente que poderiam levar à redução de gastos em desenvolvimento de produtos, capacitação gerencial e melhoria das rotinas e processos de fabricação, diminuindo assim, a habilidade da empresa em criar valor econômico futuro.

Embora, nos últimos anos tenha havido uma tendência das empresas de bens de capital buscar taxas de retorno ou a maximização de resultados financeiros, por meio da substituição da fabricação por importações, revendas de máquinas e equipamentos (REHDER, 2010), o estudo mostrou que fabricar máquinas $\mathrm{e}$ equipamentos ainda é uma atividade competitiva e distante da situação da financeirização da empresa. 
A influência da estratégia e das capabilidades para obtenção de desempenho nas empresas de bens de capital

A terceira implicação referiu-se a não evidência do impacto direto das estratégias empresas nos desempenhos operacionais e financeiros. Esta não evidência conduz à reflexão dos pressupostos básicos para o desempenho: a estrutura de competição e posicionamento de mercado ou capabilidades internas da empresa? A conexão entre as decisões que os gestores tomam e controlam e o desempenho (lucratividade) de uma empresa é mediada por um grande número de relações econômicas (BESANKO et al., 2006). Portanto, o sucesso de qualquer estratégia depende de se as decisões de uma empresa são ou não compatíveis com essas relações. Nesse sentido, Whittington (2001), argumenta que é difícil tomar decisões estratégicas, especialmente, em razão da dinâmica organizacional relacionada ao mercado. $\mathrm{O}$ resultado obtido parece evidenciar as dificuldades relacionadas às decisões estratégicas, como se pode observar pela influência das capabilidades internas no desempenho.

Assim, com as fundamentações teóricas e resultados empíricos obtidos, pode-se concluir que existe evidência, de que o desempenho nas empresas do setor de bens de capital é mais efetivo, quando ajustado à relação entre estratégia e capabilidade, mostrando assim, a importância das capabilidades operacionais internas na obtenção da vantagem competitiva.

Atividades rotineiras às empresas de bens de capital como contratação de mão de obra especializada, importação de máquinas usadas, de baixo custo e sem similar nacional, cumprimento da carga tributária, engajamento

comprometimento dos profissionais mais jovens, manutenção de estoques de sobressalentes, concorrência de produtos estrangeiros, além das altas taxas de juros e de câmbio, são atividades, muitas delas, distantes de controles administrativos da companhia, dado que, extrapolam as fronteiras da empresa. Por conta disso, manter o ajuste ou a relação positiva entre estratégias empresariais e capabilidades operacionais em manufatura é um processo altamente desafiador.

Por fim, para prosseguimento da pesquisa são sugeridos:

a) coletar dados periodicamente, mantendo a amostra fixa de empresas do setor de bens de capital, tornando a pesquisa de concepção transversal para longitudinal. Desse modo, a análise da relação entre estratégia, capabilidade e desempenho, se a conexão (ou ajuste) é causa ou consequência do desempenho se tornará mais consistente, dado que, teoria e processos de mudanças estarão presentes nas análises;

b) testar modelos alternativos em equações estruturais comparando o modelo proposto com uma série de modelos concorrentes, os quais atuam como explicações alternativas para o modelo proposto, entre os quais, considerar a capabilidade como moderador ou mediador da relação entre estratégias e desempenho. Desse modo, pode-se verificar se o modelo proposto, independentemente de ajuste geral (dentro de limites razoáveis), é aceitável, porque nenhum outro modelo analogamente formulado pode atingir um maior grau de ajuste.

\section{Referências}

ARBIX, G.; SALERNO, M. S. Política Industrial é Coisa de Rico. São Paulo: OESP - Aliás, p. J7, 11 de maio de 2008.

BACIC, M. J.; DA SILVEIRA, R. L. F.; FERREIRA DE SOUZA, M. C. A. Gestão Imprudente do Risco Financeiro como Elemento de Distribuição de Valor: Uma Reflexão a Partir do Uso de Derivativos por Empresas Lideres Brasileiras. Braga (Universidade do Minho): Revista del Instituto Internacional de Costos. v. 6, enero/junio 2010. 
BANCO NACIONAL DE DESENVOLVIMENTO ECONÔMICO E SOCIAL (BNDES). Circular $\mathbf{n}^{\mathbf{0}} \quad \mathbf{1 1 / 2 0 1 0}$. Disponível em: <http://www.bndes.gov.br/ SiteBNDES/export/sites/default/bndes_pt/ Galerias/Arquivos/produtos/download/Circ 011_10.pdf>. Acesso em: 01 Jul. 2012

BARNEY, J. Firm Resources and Sustained Competitive Advantage, Journal of Management. v. 17, n. 1, p. 99-120, 1991.

BESANKO, D.; DRANOVE, D.; SHANLEY, M.; SCHAEFER, S. A Economia da Estratégia. Porto Alegre: Bookman, 2006.

BRESSER-PEREIRA. Financeirização e riquezas fictícia. São Paulo: FSP, 23/02/2009. Disponível em: <http://ww w.bresserpereira.org.br/articles/2009/246.F inanceiriza\%C3\%A7\%C3\%A3o_e_riqueza _fict $\%$ C3\%ADcia.pdf $>$. Acesso em 1 Out. 2011.

BROWN, S; LAMMING, R; BESSANT, J; JONES, P. Administração da Produção e Operações. Rio de Janeiro: Campus / Elsevier, 2006.

BROWN, S. BLACKOMON, K. Aligning manufacturing strategy and business-level competitive strategy in new competitive environments: the case for strategic resonance. Journal of Management Studies, v. 42, n. 4, p. 793-815, 2005.

CEPEDA, G.; VERA, D. Dynamic capabilities and operational capabilities: a knowledge management perspective. Journal of Business Research, v. 60, n.5, p. 426-437, 2007.

CHASE, R. B; JACOBS, F. R.; AQUILANO, N. J. Administração da Produção para a Vantagem Competitiva. Porto Alegre: Bookman, 2006.

CHRISTOPHER, M. Logística e Gerenciamento da Cadeia de Suprimentos: Estratégia para Redução de Custos e Melhoria dos Serviços. São
Paulo: Pioneira, 1997.

CHRISTOPHER, M; TOWILL, D. An integrated model for the design of agile supply chains. International Journal of Physical Distribution \& Logistics Management, v. 31, n. 4, p. 235-246, 2001.

DEMING, W. E. Qualidade: A Revolução da Administração. Rio de Janeiro: Marques-Saraiva, 1990.

DIERICKX, I.; COOL, K. Asset Stock Accumulation and Sustainability of Competitive Advantage. Management Science, v. 35, n. 12, p. 1504-1511, 1989.

DiMAGGIO, P. J.; POWELL, W. W. A Gaiola de Ferro Revisitada: Isomorfismo Institucional e Racionalidade Coletiva nos Campos Organizacionais. São Paulo: Revista de Administração de Empresas, v. 45 , n. 2, p. 74-89, 2005

DORNIER, P. P. et al. Logística e Operações Globais. Textos e Casos, São Paulo: Atlas, 2000.

DRNEVICH, P. L.; KRIAUCIUNAS, A. P. Clarifying the conditions and limits of the contributions of ordinary and dynamic capabilities to relative firm performance, Strategic Management Journal, v. 32, n. 3, p. 254-279, 2011.

EASTERBY-SMITH，M.; THORPE，R.; LOWE, A. Pesquisa Gerencial em Administração. São Paulo: Pioneira, 1999.

FERRAZ, J. C. Precisamos de Projetos. Dinheiro não Faltará. São Paulo: OESP Economia, p. B9, 18/05/2008.

GAITHER, N.; FRAZIER, G. Administração de Produção e Operações. São Paulo: Pioneira/Thomson Learning, 2001.

GAGNON, S. Resource-based competition and the new operation strategy, International Journal of Operations \& Production Management, v. 19, n. 2, p. 125-138, 1999. 
A influência da estratégia e das capabilidades para obtenção de desempenho nas empresas de bens de capital

GREEN JR, K. W.; WHITTEN, D.; INMAN, R. A. The Impact of Logistics Performance on Organizational Performance in a Supply Chain Context. USA: Supply Chain Management: An International Journal, v. 13, n. 4, p. 317-327, 2008 .

HAIR, J. F. et al. Análise de Dados Multivariados, Porto Alegre: Bookman, 2005.

HELFAT, C. E.; WINTER, S. G. Untangling dynamic and operational capabilities: strategy for the (n)everchanging world, Strategic Management Journal. v. 32, n. 11, p. 1243-1250, 2011.

HITT, M. A.; IRELAND, R. D.; HOSKISSON, R. E. Administração Estratégica. São Paulo: International Thomson Editores, 2002.

HUNGER, J. D.; WHEELEN, T. L. Essentials of strategic management, Upper Saddle River, N.J.: Prentice-Hall, 2000.

INSTITUTO BRASILEIRO DE GEOGRAFIA E ESTATÍSTICAS (IBGE). Classificação Nacional de Atividades Econômicas - CNA. Disponível em: <http://www.ibge.gov.br/home/estatistica/e conomia/classificacoes/cnaef1.1/default.sht m> Acesso em 01 Jul. 2012 (IBGE/CNA, 2011).

JÖRESKOG， K. G.; SÖRBOM， D. LISREL 8: Users Reference Guide. USA: Scientific Software International SSI, 1993.

KAPLAN, R. S.; NORTON, D. P. Alinhamento, Utilizando o Balanced Scorecard para Criar Sinergias Corporativas. Rio de Janeiro: Campus/Elsevier, 2006.

KLINE, R. B. Principles and Practice of Structural Equation Modeling. London: The Guilford Press, 2005.

LYNCH, D. F.; KELLER, S. B.; OZMENT, J. The effects of logistics capabilities and strategy on firm performance,
Journal of Business Logistics, v. 21, p. 47-67, 2000.

MALLABY, S. É possível combinar impostos e comércio. São Paulo: OESP Economia, p. B3, 21 de fevereiro de 2007.

MEYER, J. W.; ROWAN, B. Institutionalized Organizations: Formal Structure as Myth and Ceremony In: POWELL, W.; DiMAGGIO, P.J. (Org.) The New Institutionalism in Organizational Analysis. Chicago: University of Chicago Press, p. 41-62, 1991.

MORASH, Edward A. Supply Chain Strategies, Capabilities, and Performance. Transportation Journal, v. 41, n.1, p. 3754, 2001.

MOTTA, F. C. P.; VASCONCELOS, I. F. G. Teoria Geral da Administração. São Paulo: Pioneira Thomson Learning Ltda. 2002.

PRAHALAD, C. K; HAMEL, G. The Core Competence of the Corporation, USA: Harvard Business Review. May-June 1990.

PETERAF, M. A. The cornerstones of competitive advantage: A resource-based view, Strategic Management Journal, v. 14, n. 3, p. 179-191, 1993.

PORTER, M. E. Vantagem Competitiva, Criando e Sustentando um Desempenho Superior. Rio de Janeiro: Campus, 1985.

REHDER, M. Setor de Máquinas e Equipamentos Perde Espaço no Mercado Mundial. São Paulo: OESP. Versão impressa, 8 de março de 2010.

ROSS, S. A.; WESTERFIELD, R. W.; JORDAN, B. D. Princípios de Administração Financeira. São Paulo: Atlas, 2008.

RUMELT, R. How Much does Industry Matter? Strategic Management Journal, v. 12 , n. 3, p. 167-185, 1991.

SERVIÇO BRASILEIRO DE APOIO ÀS MICRO E PEQUENAS EMPRESAS 
(SEBRAE). Anuário do Trabalho na Micro e Pequena Empresa 2010 - 2011. Disponível em: <<http://www.biblioteca.se brae.com.br/bds/bds.nsf/25BA39988A741 0D78325795D003E8172/\$File/NT000472 76.pdf>. Acesso em: 05 Jul. 2012.

SKINNER, W. Manufacturing - Missing Link in Corporate Strategy. USA: HBR, 1969.

TEECE, D. J. Explicating dynamic capabilities: the nature and microfoundations of (sustainable) enterprise performance, Strategic Management Journal, v. 28, n. 13, p. 1319-1350, 2007.

TEECE, D. J; PISANO, G; SHUEN, A. Dynamic Capabilities and Strategic Management, Strategic Management Journal, v. 18, n. 7, p. 509-533, 1997.

VERGARA, S. C. Projetos e Relatórios de Pesquisa em Administração. São Paulo: Atlas, 2009.

WARD, P. T. et al. Business environment, operations strategy, and performance: an empirical study of Singapore manufactures, Journal of Operations Management, v. 13, n. 2, p. 99-115, 1995.

WEERAWARDENA, J.; MAVONDO, F. T. Capabilities, innovation and competitive advantage, Industrial Marketing Management, v. 40, n. 8, p. 1220-1223, 2011.

WERNERFELT, B. A resource-based view of the Firm, Strategic Management Journal, v. 5, n. 2, p. 171-180, 1984.

WHITTINGTON, R. O que é Estratégia. São Paulo: Thomson, 2001.

WOOD, W. J.; ZUFFO, P. K. Supply Chain Management. Revista de Administração de Empresas, São Paulo, v. 38, n. 3, p. 55-63, julho/setembro 1998. 